DOI: 10.20472/IAC.2019.045.043

\author{
ADAM VILLA \\ Providence College, United States
}

\title{
TEACHING WITH TABLEAU: INFUSING ANALYTICS INTO YOUR COURSE
}

\begin{abstract}
:
Tableau is a software application that can help you visualize and understand your data. It can connect to almost any data type and allows users to quickly drag and drop data items to create visualizations that can be shared across platforms. Tableau can be infused into any course that works with data and several examples of data analysis in different disciplines will be demonstrated and explained. This talk will showcase some of the software's capabilities, including a variety of visualizations, tables, dashboards, and stories. It is also an excellent tool for a data analytics course and provides a great supplemental application for a database systems course, as Tableau can interface with most popular database systems. At the end of the talk, sample class exercises, and projects from an introductory data analytics course will also be discussed and presented.
\end{abstract}

\section{Keywords:}

Data Analytics, Teaching Tool, Software 